International Journal of Pure and Applied Mathematics

Volume 89 No. 2 2013, 123-139

ISSN: 1311-8080 (printed version); ISSN: 1314-3395 (on-line version)

url: http://www.ijpam.eu

doi: http://dx.doi.org/10.12732/ijpam.v89i2.1

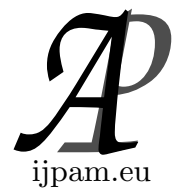

\title{
COLORING GRAPHS TO CLASSIFY SIMPLE CLOSED GEODESICS ON CONVEX DELTAHEDRA
}

\author{
Kyle A. Lawson ${ }^{1}$, James L. Parish ${ }^{2}$, \\ Cynthia M. Traub ${ }^{3}$, Adam G. Weyhaupt ${ }^{4} \S$ \\ 1,2,3,4 Department of Mathematics and Statistics \\ Southern Illinois University Edwardsville \\ Box 1653, Edwardsville, IL 62026-1653, USA
}

\begin{abstract}
We obtain a complete classification of all simple closed geodesics on the eight convex deltahedra by solving a related graph coloring problem. Geodesic segments in the neighborhood of each deltahedron vertex produce a limited number of crossing angles with deltahedron edges. We define a coloring on the edge graph of a deltahedron based on these angles, and we show that the set of graph colorings compatible with edge-colorings of the neighborhood graphs of radius one classifies all possible simple closed geodesics on all convex deltahedra.
\end{abstract}

AMS Subject Classification: 52B10, 05C15, 37D40

Key Words: polyhedra, deltahedra, geodesics, graph coloring

\section{Introduction}

A geodesic $\gamma$ on a polyhedron $\mathcal{P}$ is a locally shortest curve $\gamma: \mathbb{R} \rightarrow \mathcal{P}$. A geodesic segment is similar except that the domain is a closed, bounded interval. In each face of the polyhedron, a geodesic is a line segment. In two adjacent faces, a geodesic will form a straight line after one of the faces is rotated about their common edge by the dihedral angle. A development of a polyhedron $\mathcal{P}$ into

Received: $\quad$ May 7, 2013

(c) 2013 Academic Publications, Ltd.

${ }^{\S}$ Correspondence author url: www.acadpubl.eu 
the plane (induced by a geodesic $\gamma$ ) is a sequence of edge-adjacent polygons congruent to the faces of $\mathcal{P}$ (projected in the order by which they are crossed by $\gamma$ ). Informally, this is the image obtained by "unrolling" the surface of the polyhedron into the plane. If one develops a polyhedron into the plane, the image of a geodesic in a development will be a line. A closed geodesic is a geodesic segment $\gamma:[a, b] \rightarrow \mathcal{P}$ so that $\gamma(a)=\gamma(b)$ and so that the tangent vectors at $a$ and $b$ have the same direction (if $\gamma(a)$ and $\gamma(b)$ lie on an edge, then the tangent vectors have the same direction after rotation by the dihedral angle). A simple closed geodesic is a closed geodesic whose only self-intersection occurs at the endpoints.

The classification of simple closed geodesics on polyhedra has recently been studied by a number of authors. Galperin [6] has shown that a generic polyhedron will have no simple closed geodesics; a necessary condition for a polyhedron to have a simple closed geodesic is that its set of vertices can be partitioned into two sets, each having total curvature $2 \pi$. It is known that there are arbitrarily long closed geodesics on a regular tetrahedron; furthermore, all geodesics on a regular tetrahedron are simple. (These remarkable properties hold because the flat torus is a double cover of the tetrahedron.) Fuchs and Fuchs [4] classified the geodesics on three other Platonic solids: the cube, the octahedron (both also considered by [1]), and the icosahedron. Fuchs [5] and Lawson [7] have recently independently completed the classification of simple closed geodesics on the dodecahedron.

A deltahedron is a polyhedron with equilateral triangle faces. It is known [3] that there are exactly 8 convex deltahedra: the tetrahedron, triangular dipyramd, octahedron, pentagonal dipyramid, snub disphenoid, triaugmented triangular prism, gyroelongated square dipyramid, and the icosahedron. (We examine only properly convex deltahedra and so exclude the possibility of 6 triangles meeting at a vertex. The techniques here apply equally well to nonproperly convex deltahedra.) In this paper, we complete the classification of simple closed geodesics on the 8 convex deltahedra.

We consider in this paper edge-colorings of the edge graph formed from the collection of all vertices and edges of each deltahedron. An edge-coloring assigns to each edge of a graph one color from a finite set of colors. For our purposes, we do not seek to avoid colorings in which incident edges receive different colors. The neighborhood of radius $r$ of a vertex $v$ in a graph $G$ is the induced subgraph formed from the set of vertices including $v$ and all vertices reachable from $v$ by a path of length $r$ or less. We consider all possible neighborhood graphs of radius one from a vertex of a convex deltahedron - these are wheel graphs with one central vertex and 3,4 , or 5 outer vertices. We color these wheel graphs according 
to the possible angles a geodesic segment can make with consecutive edges it crosses, creating a (small) finite set of allowable colorings. We then consider the question of whether there exists a global edge-coloring of each deltahedron $\mathcal{P}$, such that, for every vertex of $\mathcal{P}$, the neighborhood graph of radius one receives an allowable coloring. This work is related to that of Esselstein and Winkler[2], who have studied the algorithmic complexity of building graphs with specified vertex-neighborhood colorings.

The rest of the paper is organized as follows. In Section 2, we do the necessary geometric analysis to establish the valid neighborhood graph edge colorings corresponding to the edge crossing behavior of simple closed geodesics. In Section 3 we present the valid global edge colorings constructed from the local constraints given in Section 2. We prove that each coloring gives rise to a simple closed geodesic on the specified deltahedron, and use symmetries to condense the list of valid colorings into a classification of all simple closed geodesics on convex deltahedra. We provide images of the valid edge colorings, together with an image of the corresponding geodesics.

\section{Terminology and Foundational Results}

Our proof relies on the ability to color the edges of a deltahedron based on the angle at which they are crossed by a simple closed geodesic. For this, we require the following theorem.

Theorem 1. All segments of a simple closed geodesic on a deltahedron that lie in a fixed face of the deltahedron are parallel.

Proof. Let $\gamma$ be a simple closed geodesic, and consider a face containing at least two geodesic segments $\gamma_{1}$ and $\gamma_{2}$ of $\gamma$; call the edge crossed by both segments $\overline{A B}$ and the face $\triangle A B C$. Define $\alpha_{i}$ to be the angle between $\overline{A B}$ and $\gamma_{i}$ measured counterclockwise using the inward pointing normal.

We claim that $\alpha_{1}=\alpha_{2}+\frac{k \pi}{3}(\bmod \pi)$ for some $k \in\{0,1,2\}$. To see this, develop forwards and backwards from the segment $\gamma_{1}$ until the entire geodesic $\gamma$ is developed. Since the faces crossed by the geodesic are equilateral triangles, the development will coincide with the tiling of the plane by equilateral triangles. Images of $\triangle A B C$ encountered in the development may differ by a rotation by a multiple of $\frac{\pi}{3}$, and the segment $\gamma_{2}$ will appear in some (possibly rotated) image of $\triangle A B C$ in this development. We conclude that $\alpha_{1}=\alpha_{2}+\frac{k \pi}{3}(\bmod \pi)$.

Define Corridor 1 to be the region between line $\overleftrightarrow{A C}$ and the line through $B$ parallel to $\overleftrightarrow{A C}$. Define Corridor 2 to be the region between line $\overleftrightarrow{B C}$ and 
the line through $A$ parallel to $\overleftrightarrow{B C}$. These two corridors are shown in Figure 1. We now consider two simultaneous developments of $\gamma$ into the plane (and

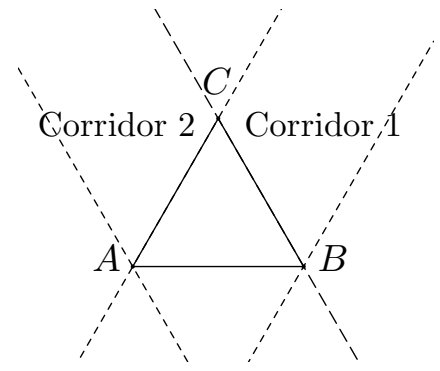

Figure 1: Two corridors in the plane; the horizontal edge $\overline{A B}$ is crossed by two geodesic segments.

into Corridors 1 and 2) by fixing an image of $\triangle A B C$ containing images of $\gamma_{1}$ and $\gamma_{2}$ and developing $\gamma$ forwards and backwards from both segments. Let $\overleftrightarrow{\gamma_{i}}$ denote the development of geodesic $\gamma_{i}$ into the plane. Since $\gamma_{i}$ is a geodesic segment, the development of $\gamma_{i}$ will be a straight line contained in corridor 1 (resp. corridor 2) until possibly the line $\overleftrightarrow{\gamma_{i}}$ leaves corridor 1 (resp. corridor 2).

Partition the set of possible values $(0, \pi)$ for angles $\alpha_{i}$ into the following intervals and singleton sets: $\left(0, \frac{\pi}{3}\right),\left\{\frac{\pi}{3}\right\},\left(\frac{\pi}{3}, \frac{2 \pi}{3}\right),\left\{\frac{2 \pi}{3}\right\}$, and $\left(\frac{2 \pi}{3}, \pi\right)$. Assume towards a contradiction that $\gamma_{1}$ and $\gamma_{2}$ are not parallel in $\triangle A B C$. Thus $\alpha_{1}=$ $\alpha_{2}+\frac{k \pi}{3}(\bmod \pi)$ for some $k \in\{1,2\}$, and so $\alpha_{1}$ and $\alpha_{2}$ are elements of different sets from the list above. Note that if $\overleftrightarrow{\gamma_{1}}$ and $\overleftrightarrow{\gamma_{2}}$ intersect in some corridor, they intersect on the polyhedron. We consider two cases. If $\alpha_{1}=\frac{l \pi}{3}$ for some $l \in\{1,2\}$, then $\alpha_{2}=\frac{(3-l) \pi}{3}$, and the lines $\overleftrightarrow{\gamma_{1}}$ and $\overleftrightarrow{\gamma_{2}}$ will cross in the intersection of Corridors 1 and 2. Otherwise, we orient the corridors by aligning $\overline{A B}$ horizontally so that the corridors inherit the orientation shown in Figure 1. If the intersection of $\overleftrightarrow{\gamma_{i}}$ with the left (resp. right) boundary of the corridor is below segment $\overline{A B}$, say that $\overleftrightarrow{\gamma_{i}}$ crosses the corridor from left to right (resp. right to left). If $\overleftrightarrow{\gamma_{1}}$ and $\overleftrightarrow{\gamma_{2}}$ cross the same corridor in different directions (one left to right and one right to left), then they must intersect in that corridor. Table 1 shows that for any choice of $\alpha_{1}$, and for any choice of corresponding value of $\alpha_{2}$, there exists a corridor in which the two developments of $\overleftrightarrow{\gamma_{1}}$ and $\overleftrightarrow{\gamma_{2}}$ have opposite orientation, and therefore intersect. Since such an intersection contradicts our initial assumption that $\gamma$ was a simple closed geodesic, we conclude that $\gamma_{1}$ and $\gamma_{2}$ are parallel. 


\begin{tabular}{r|cc} 
Angle $\alpha_{i}$ & Corridor 1 & Corridor 2 \\
\hline$\left(0, \frac{\pi}{3}\right)$ & left to right & left to right \\
$\left(\frac{\pi}{3}, \frac{2 \pi}{3}\right)$ & right to left & left to right \\
$\left(\frac{2 \pi}{3}, \pi\right)$ & right to left & right to left
\end{tabular}

Table 1: The angle $\alpha_{i}$ of geodesic $\gamma_{i}$ determines the way in which it crosses the corridors.

Since all segments of a simple closed geodesic in a face are parallel, the angle $\alpha$ between the geodesic and any edge, measured counterclockwise using the inward pointing normal, depends only on the edge. We therefore can color each edge of $\mathcal{P}$ by assigning it one letter from the set $\{U, R, H, L\}$, depending on whether the edge is uncrossed $(U)$ or whether $\alpha$ lies in the interval $\left[\frac{2 \pi}{3}, \pi\right)$, $\left[\frac{\pi}{3}, \frac{2 \pi}{3}\right)$, or $\left(0, \frac{\pi}{3}\right)$ respectively. Angle considerations immediately yield the following:

Corollary 2. Either every edge is uncrossed in a face, or exactly one edge of the face is colored $H$ and another is colored $L$ or $R$. If one edge of a face is colored $L$, then the edge of that face located immediately counterclockwise along the boundary of the face must be colored $H$; similarly, if an edge is colored $R$ then the edge immediately clockwise along the boundary of the face must be colored $H$.

Every geodesic on a deltahedron can be developed into the plane so that the image of the deltahedron's vertices lie in the lattice generated by 1 and $\zeta=e^{i \pi / 3}$. Let $\gamma$ be a closed geodesic on a deltahedron $\mathcal{P}$ passing through an edge $\overline{A B}$. Since $\gamma$ is a geodesic, some development of $\gamma$ into the plane will be a line segment bounded at each end by an image of $\overline{A B}$. Now, since the geodesic $\gamma$ is closed, the difference $\lambda$ between the endpoints of this line segment will lie in the lattice. $\lambda$ is called a generator of $\gamma$ with respect to edge $\overline{A B}$. It is clear that each closed geodesic determines a unique generator.

We now describe how to determine the generator of a geodesic by only knowing how the geodesic alternates among edges. Fix an edge of $\mathcal{P}$ colored $H$ (such an edge must exist since every face crossed by $\gamma$ must contain an edge colored $H$ ). We consider the sequence of edge colors corresponding to the list of crossings made sequentially by $\gamma$. Angular considerations imply that the next two edge crossings by $\gamma$ are colored either $R, H$ or $L, H$, as illustrated in Figure 2 .

We abbreviate the ' $\mathrm{RH}$ ' sequence as + and the ' $\mathrm{LH}$ ' sequence as -. Since this two-face process ended on an edge colored $H$, we can continue coloring in 

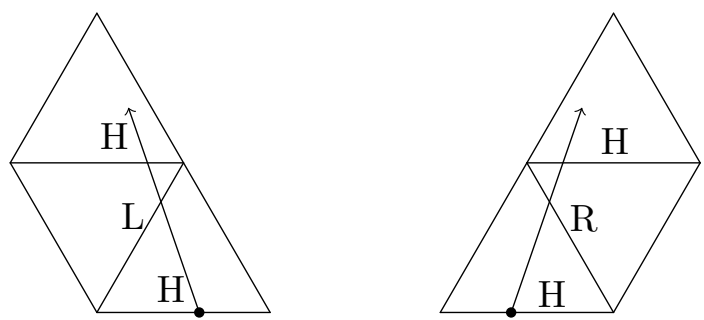

Figure 2: An edge crossing colored ' $\mathrm{H}$ ' implies that the next two crossings are either 'LH' or 'RH'.

this fashion to obtain a geodesic turn sequence of + and - associated to $\gamma$ and to the initial edge. Changing the initial edge results in a cyclic permutation of the sequence of $+/-$.

The next definition and proposition pertain to geodesic segments; the result is an important tool in the proof of Theorem 6 .

Definition 3. Let $\gamma$ be a geodesic segment. Define $m$ to be the sum of the number of + and - in the geodesic turn sequence, and define $n$ to be the number of - . The signature of $\gamma$ is the pair $(m, n)$. The increment of $\gamma$ is $\Delta x / \Delta y$ in the coordinate system given by -1 and $\zeta$.

Proposition 4. If a geodesic segment has signature $(m, n)$, then the increment of $\gamma$ is strictly between $\frac{n-1}{m}$ and $\frac{n+1}{m}$.

Proof. Since the signature is $(m, n)$, the geodesic intersects $m$ horizontal lines in addition to the initial edge, so $\Delta y=m$. Similarly, the geodesic crosses exactly $n$ lines parallel to the vector $\zeta$, so $n-1<\Delta x<n+1$.

If $\gamma$ is a simple closed geodesic, the increment of any segment of $\gamma$ is the same as the increment of $\gamma$. However, two segments may have different signatures that provide different bounds on the increment.

Lemma 5. If the generator of a closed geodesic $\gamma$ is $m \zeta-n$, where $m$ and $n$ are integers, then $m$ is the sum of the number of + and - in the geodesic turn sequence, while $n$ is the number of - . The signature of $\gamma$ is the pair $(m, n)$ and the increment of $\gamma$ is $\frac{n}{m}$.

Proof. Since each 'LH' or 'RH' sequence takes the geodesic from a horizontal line to a horizontal line, $m$ is the sum of the number of + and - in the geodesic turn sequence. Also, each 'LH' pair will necessarily cross exactly one of the 
lines parallel to the vector $\zeta$, so $n$ is the number of - . The statements about signature and increment are immediate consequences.

Theorem 6. Let $\gamma$ be a simple closed geodesic on a deltahedron $\mathcal{P}$. If $\mathcal{P}$ has no vertices of degree 3 , then $\gamma$ crosses each edge at most once.

Proof. Assume by way of contradiction that $\mathcal{P}$ has no vertices of degree 3 and that $\gamma$ crosses some edge more than once. Since $\gamma$ is a simple closed geodesic, $\gamma$ divides $\mathcal{P}$ into two regions each with curvature $2 \pi$. There is some face so that $\gamma$ crosses all three edges; if not, then one of the regions would contain no vertices which violates the curvature condition. In this face, the vertex $X$ between the segments must either have degree 4 or degree 5 .
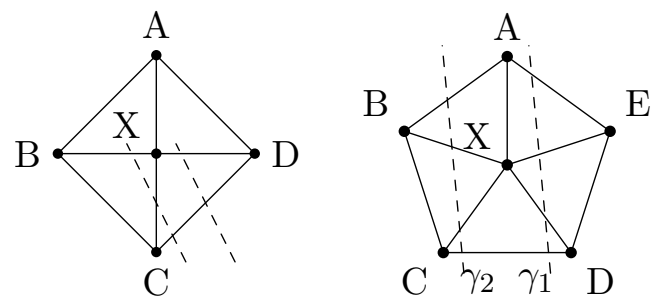

Figure 3: If a simple closed geodesic crosses an edge more than once, there must be degree 3 vertices.

For the remainder of this proof we refer to Figure 3. Suppose that $X$ has degree 4 . The edges of $\triangle X C D$ must be colored so that $\overline{X C}$ is colored $L, \overline{C D}$ is colored $H$, and $\overline{X D}$ is colored $R$. Since $\overline{X C}$ is colored $L, \overline{X B}$ must be colored $H$; similarly, $\overline{X A}$ must be colored $H$ by Corollary 2. However, this causes $\triangle X A B$ to have two edges colored $H$; a contradiction.

If instead $X$ has degree $5, \triangle X C D$ is colored $L, H$, and $R$. Then $\overline{X B}$ is colored $H$, and $\overline{X E}$ is colored $H . \overline{X A}$ can be colored neither $L$ nor $R$ since either $\triangle X A B$ or $\triangle X E A$ would have two sides colored $H$. Therefore, $\overline{X A}$ is uncrossed and so $\overline{A B}$ is $L$ and $\overline{A E}$ is $R$. Call these two geodesic segments $\gamma_{1}$ and $\gamma_{2} ; \gamma_{1}$ has signature $(2,0)$ and $\gamma_{2}$ has signature $(2,2)$. Now, the increment of $\gamma_{1}$ is equal to the increment of $\gamma_{2}$ because they are segments of the same closed geodesic $\gamma$. However, by Proposition 4, $\gamma_{1}$ bounds the increment of $\gamma$ strictly between $-1 / 2$ and $1 / 2$ while $\gamma_{2}$ bounds the increment of $\gamma$ strictly between $1 / 2$ and $3 / 2$; these bounds are incompatible.

Finally, the main ingredient of our proof is that there is only a small number of possible configurations around a degree four or degree five vertex: 


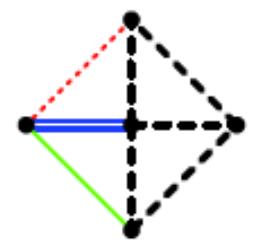

(1) $4 \mathrm{a}$

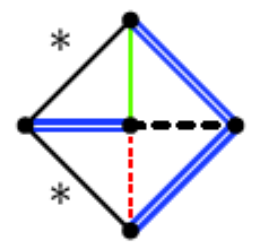

(5) $4 \mathrm{~d}$

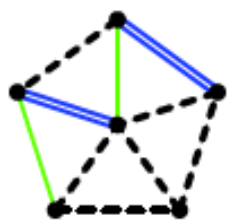

(9) $5 \mathrm{cr}$

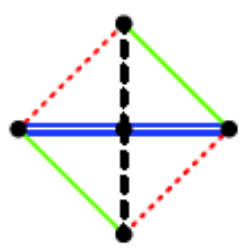

(2) $4 \mathrm{~b}$

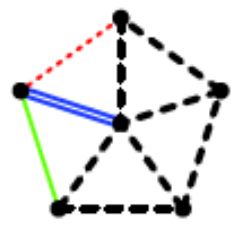

(6) $5 \mathrm{a}$

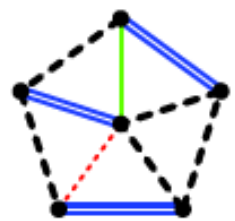

(10) $5 d$

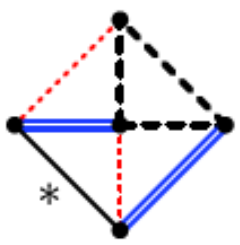

(3) $4 \mathrm{cl}$

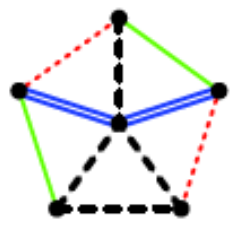

(7) $5 \mathrm{~b}$

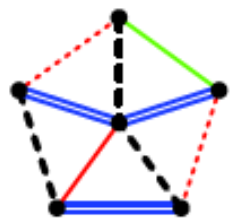

(11) $5 \mathrm{el}$

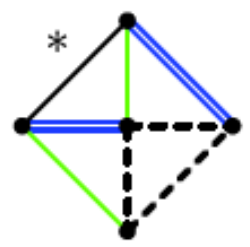

(4) $4 \mathrm{cr}$

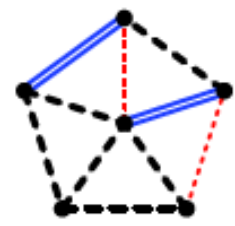

(8) $5 \mathrm{cl}$

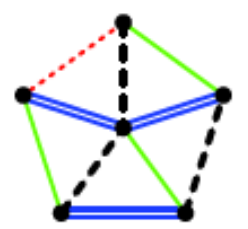

(12) $5 \mathrm{er}$

Figure 4: The possible edge crossing configurations around degree 4 and 5 vertices. Red (dotted) edges are colored ' $\mathrm{L}$ ', blue (double) edges ' $\mathrm{H}$ ', green (single) edges ' $R$ '. Edges marked with an asterisk are inconclusive on a triangular dipyramid; otherwise those edges are uncrossed. Two additional diagrams, with all edges uncrossed, are possible.

Theorem 7. Let $\gamma$ be a simple closed geodesic on a convex deltahedron. The coloring induced by $\gamma$ around degree 4 and degree 5 vertices is one of the colorings indicated in Figure 4.

Proof. We consider first the cases for both degrees four and five where only 0,1 , or 2 edges incident to the central vertex are crossed; in what follows we use the notation from Figure 3. If no edge incident to $X$ is crossed, then the only possibility is $4 \mathbf{e}$ or a similar diagram for the degree 5 case (which only occurs on 
the icosahedron). If only one edge incident to $X$ is crossed, then the remaining edges incident to $X$ are uncrossed. The crossed edge can be colored neither $L$ nor $R$ since either of these would force another of the edges adjacent to $X$ to be colored $H$. Therefore, the crossed edge must be colored $H$; without loss of generality we let the crossed edge be $\overline{X B}$. The remaining edges are forced as shown in $\mathbf{4 a}$ and $\mathbf{5 a}$ by Corollary 2 .

If two edges are crossed, those edges are either adjacent or not. If the two crossed edges are not adjacent, these must both be colored $H$, since otherwise a third adjacent edge would also be colored. The remaining edges are forced by Corollary 2 and the resulting diagrams are $\mathbf{4} \mathbf{b}$ and $\mathbf{5} \mathbf{b}$. If the crossed edges are adjacent, one edge must be $H$ and one must be either $L$ or $R$. If $X$ has degree four, then Corollary 2 forces all but one edge color and results in diagrams $\mathbf{4 c l}$ and $4 \mathbf{c r}$. If $X$ has degree five then Corollary 2 forces all of the remaining edges and yields diagrams $\mathbf{5 c l}$ and $\mathbf{5 c r}$.

Now we consider possibilities when $X$ has degree four and more than 2 edges are crossed. One edge must be horizontal; again assume $\overline{X B}$ is colored $H$. This implies that $\overline{X D}$ is uncrossed, because if $\overline{X D}$ were $H$ then both $\overline{X A}$ and $\overline{X C}$ would be uncrossed because of the competing demands placed by the two edges colored $H$, and $\overline{X D}$ cannot be either $L$ or $R$ since that would force a triangle to have two edges colored $H$. Therefore, it is not possible to have all four edges crossed. The only possibility is for $\overline{X A}$ to be $R$ and $\overline{X C}$ to be $L$. While this forces $\overline{A D}$ and $\overline{C D}$ to be $H$, the remaining two edges are undetermined; this is the case $\mathbf{4 d}$.

If $X$ has degree five and more than 2 edges are crossed, then at most two of them can be colored $H$ (no two edges colored $H$ can be adjacent). If exactly one edge is colored $H$, we assume it is $\overline{X B}$. Then $\overline{X A}$ is colored $R$ and $\overline{X C}$ is colored $L$; the remaining edges must be uncrossed, and this is case $\mathbf{5 d}$. If two edges are colored $H$, by symmetry they are $\overline{X B}$ and $\overline{X E}$. $\overline{X A}$ must be uncrossed because the $H$ colors put conflicting demands on $\overline{X A}$. We can then have either $\overline{X C}$ colored $L$ or $\overline{X D}$ colored $R$ (these are cases 5el and 5er). Since at least one edge in every face is uncrossed, we can not have both $\overline{X C}$ and $\overline{X D}$ colored.

Thus we have established the allowable colorings of neighborhood graphs of radius one from deltahedron vertices of degree four or five. Finally, we observe that some generators of geodesics are equivalent under symmetry.

Lemma 8. Consider a simple closed geodesic with generator $m \zeta-n$. After a reflection in a symmetry plane perpendicular to a horizontal edge, the geodesic is generated by $m \zeta-(m-n)$. 
Note that after a reflection in such a symmetry plane, the sequence of edge colors corresponding to the geodesic is the same except that each " $\mathrm{L}$ " becomes an " $R$ " and vice versa.

\section{Proofs of Main Theorems}

In the previous section we showed that every simple closed geodesic induces a coloring of the edges of a convex deltahedron $\mathcal{P}$ with the colors $U, L, H$, and $R$. Sufficiently small parallel translations of a simple closed geodesic will preserve both the sequence of edge crossings and the lack of self-intersections. This paper accomplishes the goal of classifying, up to symmetry and vertex-avoiding parallel translations, the simple closed geodesics on deltahedra. (A parallel translation of a geodesic is called $\gamma$ vertex-avoiding if it does not move $\gamma$ past a vertex in the planar development of $\gamma$. If a parallel translation sweeps the geodesic past a vertex, the sequence of edge crossings will change and so will the $+/-$ geodesic turn sequence.)

\begin{tabular}{l|l|l}
\begin{tabular}{l|l} 
Name \\
(Number of SCG)
\end{tabular} & Lengths & Generators \\
\hline $\begin{array}{l}\text { tetrahedron ( } \infty) \\
\text { triangular dipyramid (1) }\end{array}$ & $2 \sqrt{3}$ & $4 \zeta-2$ \\
octahedron (2)[4] & $3,2 \sqrt{3}$ & $3 \zeta, 4 \zeta-2$ \\
pentagonal dipyramid (1) & $2 \sqrt{3}$ & $4 \zeta-2$ \\
triagumented triangular & $4, \sqrt{19}$ & $4 \zeta, 5 \zeta-2$ \\
prism (2) & & \\
snub disphenoid (5) & $4,2 \sqrt{3}, 2 \sqrt{7}, \sqrt{13}$, & $4 \zeta, 4 \zeta-2,3 \zeta-1$, \\
& $\sqrt{19}$ & $4 \zeta-1,5 \zeta-2$ \\
gyroelongated & $4,2 \sqrt{7}, \sqrt{19}, \sqrt{21}$ & $4 \zeta, 6 \zeta-2,5 \zeta-2$, \\
dipyramid (4) square & & $5 \zeta-1$ \\
icosahedron (3) [4] & $5,3 \sqrt{3}, 2 \sqrt{7}$ & $5 \zeta, 6 \zeta-3,6 \zeta-2$
\end{tabular}

Table 2: Simple closed geodesics on the 8 convex deltahedra.

Table 2 lists the number of simple closed geodesics on each deltahedron, the lengths of each geodesic, and the generator for each geodesic. In this section we verify this table by completing the following three steps:

1. Analyze which global edge colorings of deltahedra are compatible with 
the allowable local colorings in Figures 4 and 4. (The existence of an edge coloring is a necessary condition for a simple closed geodesic to exist.)

2. Determine the generator corresponding to the geodesic turn sequence associated to each allowable global coloring.

3. Check that the generator corresponds to a geodesic by ensuring that the development given by the coloring contains a line segment given by the generator.

In the figures that follow, we provide the possible global edge colorings allowed by the local conditions established in Section 2. Algorithmically, we begin at a degree four vertex, and proceed to generate all colorings of the edge graph of the deltahedron that use the allowable subgraph colorings. Some initial choices led to immediate coloring contradictions, while others allowed for one or more possible colorings around vertices whose edges were partially colored by previous selections. The cases we present have been simplified to account for the multiplicity given by the symmetries of the deltahedra. If a coloring can be completed, then the generator corresponding to the coloring is a consequence of Lemma 5; these generators are indicated in the figures as well. Some colorings that could not be completed could have been discarded at an earlier point in the algorithm by keeping track of curvature: the partial colorings forced the vertices to be partitioned into two sets that violate the criterion that each set must contain vertices of total curvature exactly $2 \pi$.

We finally must check that the generator corresponds to a geodesic by ensuring that the development given by the coloring contains a line segment given by the generator. We call such a coloring compatible with its generator. In all of the cases we consider, the generator and the coloring are compatible; we do not know whether this is the case more generally or only in the case of the convex deltahedra.

We now describe how to determine which colorings are compatible with a given generator. Assume that a generator $m \zeta-n$ is primitive, that is, assume that that $\operatorname{gcd}(m, n)=1$. A geodesic must connect corresponding points on a given horizontal line segment and its translation by $m \zeta-n$. This determines a parallelogram (shaded in gray in Figure 5) that contains exactly $m-1$ vertices. There are $m$ different $+/$ - sequences possible. (By sweeping a line segment parallel to the left edge of the quadrilateral from left to right, the $+/-$ sequence will change each time a vertex is crossed.) Some adjacent +- pair (namely, if the vertex being passed over is on the $k$ th horizontal, then the symbols in positions $k$ and $k+1$ will be transposed) will change to -+ or vice versa. 
For the generators below, this results in a cyclic permutation of the previous sequence. If $\operatorname{gcd}(m, n)=1$, then there are only $m$ possible sequences. We are working with a relatively small set of generators, and so it is easy to write down all of the possible sequences; they are shown in Table 3.

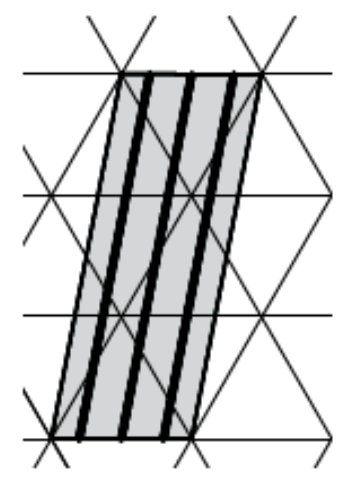

Figure 5: The three possible geodesics which determine different $+/-$ geodesic turn sequences corresponding to generator $3 \zeta-1$ are shown by the 3 dark line segments; the turn sequences are,-+++-+ , and ++- . Each is a cyclic permutation of the others. A geodesic for a given generator exists only if the $+/-$ turn sequence for the generator appears in this list.

\begin{tabular}{ll||ll} 
Generator & Turn Sequence & Generator & Turn Sequence \\
\hline$\zeta$ & + & $4 \zeta-1$ & -+++ \\
$2 \zeta-1$ & -+ & $5 \zeta-1$ & -++++ \\
$3 \zeta-1$ & -++ & $5 \zeta-2$ & -+-++
\end{tabular}

Table 3: Each generator is compatible only with cyclic permutations of the geodesic turn sequence shown.

\subsection{Triangular Dipyramid}

This is the only deltahedron we consider in this paper that contains degree three vertices. To search for valid colorings, we start with an initial vertex chosen arbitrarily from one of the degree four vertices. The choice of a local coloring from those allowed in Figure 4 specifies all edge colors with the exception of one edge. We consider two orientations of each valid coloring, as a degree four 
vertex is alternatingly adjacent to vertices of degrees three and four. Only one global coloring exists, as displayed with the corresponding geodesic in Figure 6. Since up to symmetry there is only one possible coloring of the triangular dipyramid, it follows that there is at most one simple closed geodesic. To see that there is exactly one, we need to ensure that the development defined by the coloring contains the line segment defined by the generator. The $+/-$ sequence determined by the coloring is +-+- ; this is compatible with the related primitive generator $2 \zeta-1$.
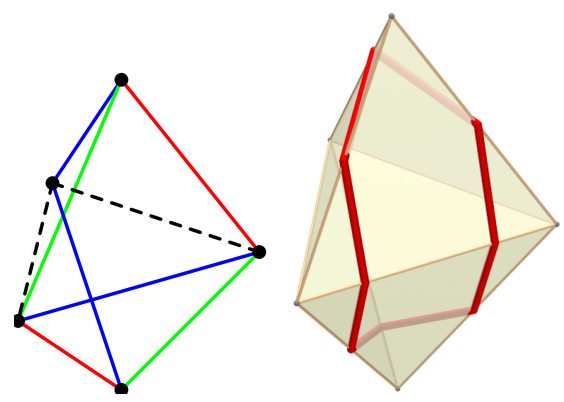

Figure 6: The only possible edge coloring of the triangular dipyramid, together with a rendering of the corresponding geodesic.

\subsection{Pentagonal Dipyramid}

All degree 4 vertices are identical, up to a rotational symmetry. Since each is incident to two apical and two equatorial edges, we must distinguish the orientation of the allowable local colorings. We denote the orientation as $\mathbf{4 a}$ (apical) or $4 \mathbf{a}$ (equatorial) depending on whether the apical or equatorial edges are colored $H$. Choices for coloring the initial vertex as $\mathbf{4 a}$ (equatorial), $\mathbf{4 b}$ (apical), or $\mathbf{4 d}$ (equatorial) all lead to valid global colorings. Each is symmetric to the others by a rotation of the dipyramid about the axis connecting the apices. Therefore, up to symmetry, there is one simple closed geodesic on the pentagonal dipyramid, corresponding to the primitive generator $2 \zeta-1$. The edge coloring is illustrated together with an image of the geodesic in Figure 7.

\subsection{Triaugmented Triangular Prism}

All individual edges incident to a degree four vertex are symmetric to one another, yet there are two types of faces incident to the degree 4 vertices. Thus, for some vertex configurations, we will again need to consider different orienta- 


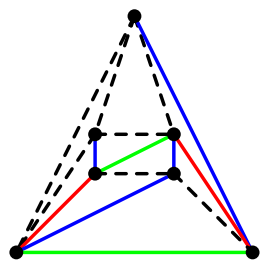

(1) $4 \zeta-2$

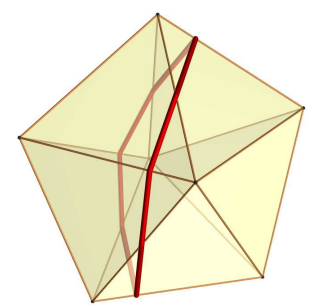

(2) $4 \zeta-2$ (red)

Figure 7: The pentagonal dipyramid has one simple closed geodesic.

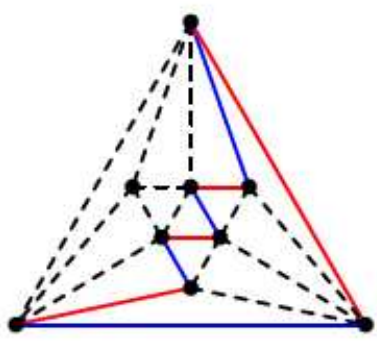

(1) $4 \zeta$

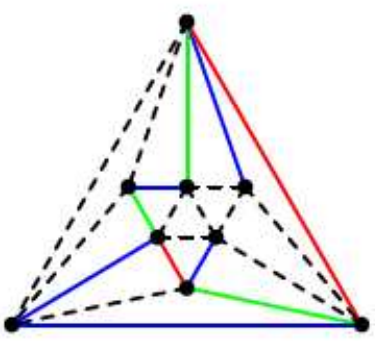

(2) $5 \zeta-2$

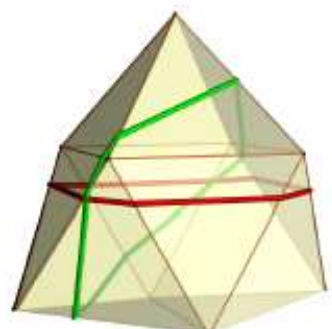

(3) $4 \zeta$ (red) and $5 \zeta-$ 2 (green)

Figure 8: The triaugmented triangular prism has two simple closed geodesics.

tions. There are two distinct global colorings, up to reflection and rotational symmetries of the triaugmented triangular prism. The corresponding generators are $5 \zeta-2$ and $4 \zeta$, illustrated in Figure 8 together with an image of the geodesics.

\subsection{Snub Disphenoid}

There are three different types of edges incident to degree four vertices, which requires us to consider multiple orientations of how to color the neighborhood of the initial vertex. After reflection and rotational symmetries (see Lemma 8), five possible colorings are obtained, corresponding to generators $4 \zeta-1,4 \zeta-2$, $5 \zeta-2,4 \zeta$, and $3 \zeta-1$. These are illustrated in Figure 9. 


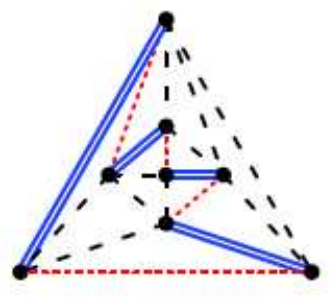

(1) $4 \zeta$

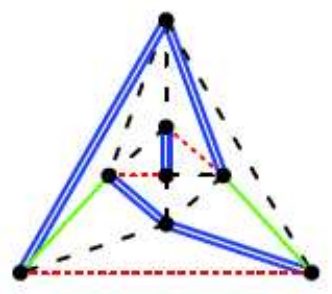

(4) $5 \zeta-3$

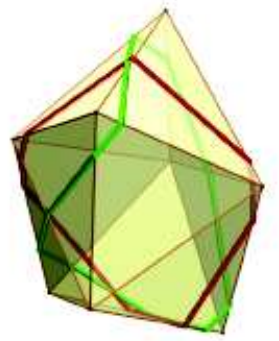

(7) $5 \zeta-3$ (red) and $6 \zeta-2$ (green)

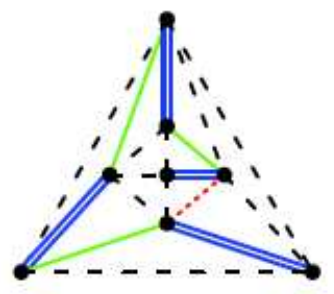

(2) $4 \zeta-1$

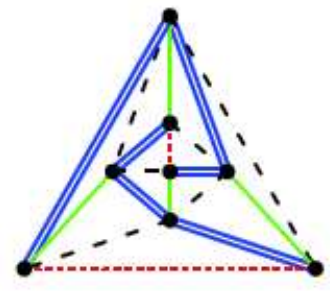

(5) $6 \zeta-2$

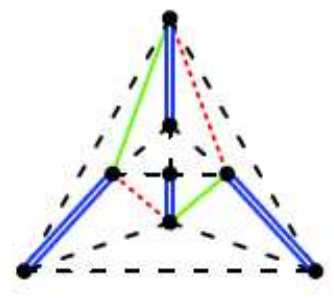

(3) $4 \zeta-2$

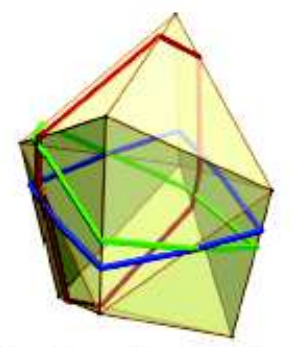

(6) $4 \zeta$ (red), $4 \zeta-1$ (green), and $4 \zeta-2$ (blue)

Figure 9: The five valid colorings of the snub disphenoid. The color of an edge describes the angle between a geodesic and that edge: red (dotted) is ' $\mathrm{L}$ ', blue (double lines) is ' $\mathrm{H}$ ', green (single line) is ' $\mathrm{R}$ ', and black (dashed) is uncrossed.

\subsection{Gyroelongated Square Dipyramid}

The symmetries of our final deltahedron simplify the colorings of degree four vertex neighborhoods, as there is only one type of edge (resp., face) incident 
to the degree four vertices. Up to symmetry, we obtain four valid colorings, corresponding to the generators $5 \zeta-2,5 \zeta-1,6 \zeta-2$, and $4 \zeta$. One can easily check that the sequences corresponding to these colorings are compatible with the generator. Images of these colorings are given in Figure 10.

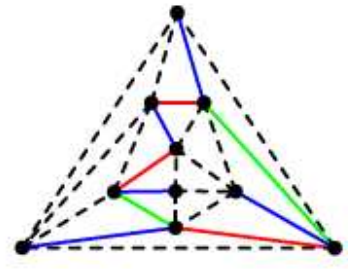

(1) $5 \zeta-3$

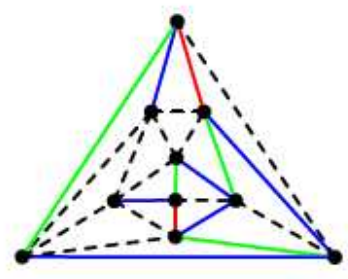

(3) $6 \zeta-2$

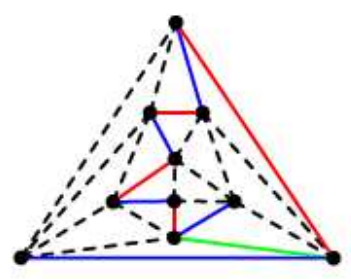

(2) $5 \zeta-4$

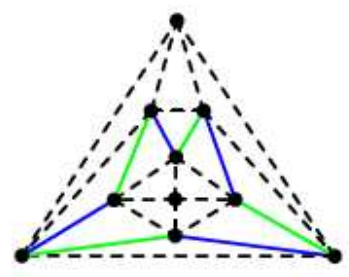

(4) $4 \zeta$

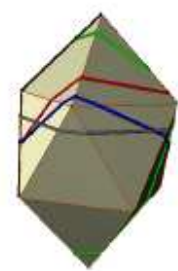

(5) $4 \zeta$ (grey), $5 \zeta-4$ (red), $6 \zeta-2$ (green), and $5 \zeta-3$ (blue)

Figure 10: The gyroelongated square dipyramid has four simple closed geodesics.

\subsection{Conclusions and Future Work}

For the convex deltahedra considered in this paper, if the local coloring constraints could be extended to a global coloring, then we found a simple closed geodesic that satisfied the given edge crossing constraints. In our initial search for these simple closed geodesics, we took heavily into account the curvature restrictions implied by Gauss-Bonnet. Some cases were ruled out initially on the grounds of curvature by forcing a vertex partition with one part having curvature greater than $2 \pi$. However, each of these was shown to eventually give rise to a coloring contradiction. A few questions remain:

- Can these edge colorings be used to classify geodesics on non-convex delta- 
hedra?

- Does there exist a deltahedron that can be edge colored, satisfying local conditions on degree four and five vertices, but that does not admit a geodesic realizing the given crossing pattern?

- Can this coloring technique be extended to classify simple closed geodesics on other polyhedra?

\section{References}

[1] A. Cotton, D. Freeman, A. Gnepp, T. Ng, J. Spivack, C. Yoder, The isoperimetric problem on some singular surfaces, J. Aust. Math. Soc., 78 No. 2 (2005), 167-197, doi: 10.1017/S1446788700008016.

[2] R. Esselstein, P. Winkler, Building graphs from colored trees, Electron. J. Combin., 17 No. 1 (2010), Research Paper 161, 18.

[3] H. Freudenthal, B.L. v. d. Waerden, On an assertion of Euclid, Simon Stevin, 25 (1947), 115-121.

[4] D. Fuchs, E. Fuchs, Closed geodesics on regular polyhedra, Mosc. Math. J., 7, No. 2 (2007), 265-279.

[5] D. Fuchs, Geodesics on a Regular Dodecahedron, Preprint, Max Planck Institute für Mathematik, Bonn (2009).

[6] G. Galperin, Convex polyhedra without simple closed geodesics, Regul. Chaotic Dyn., 8, No. 1 (2003), 45-58.

[7] K. Lawson, Personal Communication (2009).

[8] V. Protasov, Closed geodesics on the surface of a simplex, Mat. Sb., 198, No. 2 (2007), 103-120, doi: 10.1070/SM2007v198n11ABEH003900. 
